\title{
TWO DIMENSIONAL DECOMPOSITION OF THE LUMINOSIT' DISTRIBUTION OF THE SPIRAL GALAXIES : ESO598-G009, NGC1515 AND NGC7456
}

\author{
YOUNG-JUN CHOI ${ }^{1}$, BYEONG-GON PARK ${ }^{1,2}$, TAE SEOG YOON ${ }^{1}$ AND \\ HONG BAE ANN ${ }^{3}$ \\ 1 Kyungpook National University, Korea \\ 2 BOAO, Korea Astronomy Observatory, Korea \\ AND \\ ${ }^{3}$ Pusan National University, Korea
}

\section{Purpose of the two dimensional luminosity decomposition}

Because it is inevitable to lose some information in the process of one dimensional luminosity profile extraction, we developed a two dimensional luminosity decomposition method in order to examine the structure of galaxy such as bulge, bar and spiral arms.

\section{Numerical program}

We assumed de Vaucouleurs' law and exponential law for the luminosity distribution of bulge and disk, respectively. The intensity profiles for bulge and disk can be described as;

$$
\begin{array}{r}
I_{B}(r, \theta)=I_{e} 10^{\alpha(\theta)\left[\left(r / r_{e}\right)^{1 / 4}-1\right]} \\
I_{D}(r, \theta)=I_{0} \exp \left(-\frac{r}{h_{R}(\theta)}\right) \\
r=\sqrt{x^{2}+\left(\frac{y}{\cos i}\right)^{2}}, \quad \theta=\tan ^{-1}\left(\frac{y}{x}\right)
\end{array}
$$

where $r$ is distance from the center and $\theta$ is angle measured from the major axis. Using the numerical program we can determine five unknown param- 


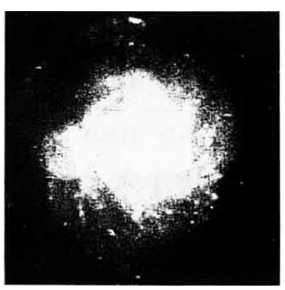

(a)

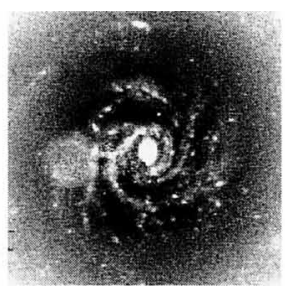

(b)

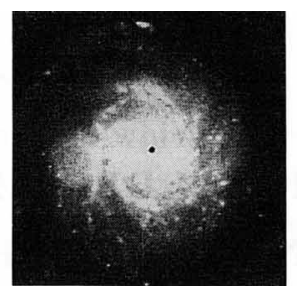

(c)

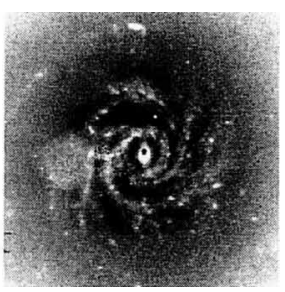

(d)

Figure 1. (a)observed image for NGC3344,(b)disk subtracted image,(c)bulge subtracted image, and (d)bulge and disk subtracted image. Because of very small bulge, (c) is not so different from (a). However, (b) and (d) show that NGC3344 has several large spiral arms, small elongated bulge component and inner ring-like struture.

eters in equations (1) to (3), denoted as $I_{e}, \alpha(\theta), r_{e}$ for bulge and $I_{0}, h_{R}(\theta)$ for disk. We took the convergence criterion as $10^{-4}$ for each value.

\section{Application to the observed galaxies}

We applied our program to the galaxies, ESO598-G009, NGC1515 and NGC7456 observed with 1-m reflector at the MSSSO, Australia. However we found that the values of the photometric parameters diverged, mainly due to high inclination. Thus we applied it to the five spiral galaxies with lower inclination angles ( $\mathrm{i}<40$ degrees) chosen from Frei et al.(1996). We could distinguish nonspherical bulges as well as spiral arms in NGC3147 and NGC3344(see Figure 1), and bar structure in NGC4394, NGC4548 and NGC5850.

\section{Difficulties and solutions}

Since galaxies have bulges with various shapes; oblate, prolate and triaxial ones, successful bulge fitting is quite difficult. This difficulty might be solved by considering projection effect of bulge shape. If we consider bar as a third component, the luminosity model for the bar is also required.

\section{Acknowledgements}

We thank Dr. R.S. deJong for helpful discussion. YJC and TSY appreciate Kyungpook Naitional University supporting travel expenses. YJC also thanks the IAU LOC for the financial support.

\section{References}

Frei, Z., Guhathakurta, P., and Gunn, J. E. 1996, $A J, 111,174$ 